\title{
Evaluation of hydrogen dissolved in rubber materials under high-pressure exposure using nuclear magnetic resonance
}

\begin{abstract}
Hirotada Fujiwara $^{1}$ and Shin Nishimura ${ }^{1,2}$
Hydrogen molecules dissolved in rubber due to high-pressure hydrogen gas exposure have been analyzed using ${ }^{1} \mathrm{H}$ nuclear magnetic resonance (NMR) with solution and solid-state probes. To analyze the characteristics of dissolved hydrogen in rubber materials and to compare them with gaseous phase hydrogen, we measured the NMR spectra of gaseous phase hydrogen and evaluated the chemical shifts and pressure dependency. Measurement using a sealed tube and liquid phase probes enabled the simultaneous analysis of dissolved hydrogen in rubber and gaseous phase hydrogen eliminated from the rubber. Two peaks at 4.3 and 4.8 p.p.m. were observed in the ${ }^{1} \mathrm{H}$ MAS NMR of the rubber samples after high-pressure hydrogen exposure. Using information from the chemical shift of the free hydrogen gas and the time dependency of hydrogen quantification in the rubber, both of these peaks were confirmed to be attributable to dissolved hydrogen. Their differences in relaxation time confirmed that their mobilities were different. In conclusion, the hydrogen dissolved in acrylonitrile butadiene rubber exists in two different forms with different mobilities. The ratio of those two hydrogens differs and is affected by the exposure pressure and
\end{abstract} elimination process time.

Polymer Journal (2012) 44, 832-837; doi:10.1038/pj.2012.111; published online 27 June 2012

Keywords: acrylonitrile butadiene rubber (NBR); gaseous; gaseous phase NMR; high-pressure gas; hydrogen; solid-state nuclear magnetic resonance (NMR)

\section{INTRODUCTION}

Progress in solid-state nuclear magnetic resonance (NMR) technology has enabled the precise analysis of polymeric materials in the solid state. It is well known that NMR provides a wide range of important information for the characterization of chemical structures as well as its quantitative evaluation, dynamics of molecular chains and intermolecular interaction.

In contrast, NMR measurement of gaseous phase materials has generally been difficult owing to its low density. The sensitivity of gaseous phase NMR measurements has been improved to obtain higher resolution spectra of gaseous phase materials despite its low density. ${ }^{1}$ There are several reports on the evaluation of gas absorbed in nanostructures using NMR and ${ }^{129} \mathrm{Xe}$ as a probe molecule for the characterization of zeolite ${ }^{2,3}$ or polymers. ${ }^{4-8}$ Hydrogen gas was found to have been trapped in absorption materials, such as fullerene ${ }^{9}$ and carbon nanotubes. ${ }^{10}$

NMR measurement is more suitable for hydrogen gas than for other gases, such as $\mathrm{CO}_{2}$, because of its high natural abundance, which is the target for evaluation. However, a high density of the sample is required, and it is difficult to build experimental systems that handle high-pressure hydrogen gas. Furthermore, in the case of polymer or clathrate samples with organic materials, it is necessary to distinguish the spectrum of gaseous hydrogen molecules from hydrogen in the chemical structure of the material.

NMR measurement with high-speed magic angle sample spinning (MAS) in a high magnetic field will allow us to obtain high-resolution spectra useful for the evaluation of hydrogen gas.

The progress in NMR technology has helped us to understand the structure and dynamics of mixtures containing polymeric materials and volatile low molecular weight compounds. For example, we have previously reported that hydrogen molecules were dissolved in rubber materials used in high-pressure hydrogen gas seals. ${ }^{11}$ Hydrogen storage cylinders used as fuel storage vessels for fuel cell electric vehicles are required to be resistant to high-pressure hydrogen gas due to their minimal dimension and weight. The rubber seals in highpressure vessels and piping devices for the hydrogen fuel storage and delivery systems are repeatedly exposed to high-pressure hydrogen gas. When rubber materials are exposed to high-pressure hydrogen gas, explosive failure by decompression could occur due to hydrogen gas that dissolves into the material under high-pressure hydrogen gas exposure. ${ }^{12}$

${ }^{1}$ Research Center for Hydrogen Industrial Use and Storage (HYDROGENIUS), National Institute of Advanced Industrial Science and Technology (AIST), Fukuoka, Japan and 2Department of Mechanical Engineering, Faculty of Engineering, Kyushu University, Fukuoka, Japan

Correspondence: Dr H Fujiwara, Research Center for Hydrogen Industrial Use and Storage (HYDROGENIUS), National Institute of Advanced Industrial Science and Technology (AIST), 744 Motooka, Nishi-ku, Fukuoka-city, Fukuoka 819-0395, Japan.

E-mail: fujiwara-hiro@aist.go.jp

Received 1 February 2012; revised 9 April 2012; accepted 17 April 2012; published online 27 June 2012 
We have previously evaluated the influence of hydrogen in rubber after high-pressure hydrogen exposure, with the aim of developing high-pressure hydrogen-resistant materials. ${ }^{13-16}$

We observed that hydrogen, an inactive and reductive gas, did not affect the chemical structure of acrylonitrile butadiene rubber (NBR), based on NMR, IR and Raman results. ${ }^{17}$ Consequently, it was suggested that explosive failure by decompression was not caused by chemical structure change but by hydrogen dissolved in the materials. Therefore, it is important to analyze the characteristics and quantity of dissolved hydrogen to understand the explosive failure by decompression mechanism or swelling behavior of rubber materials. Thus, NMR is useful for this purpose. In this paper, the results of a detailed evaluation using ${ }^{1} \mathrm{H}$ NMR for the direct observation of characteristics and quantity of dissolved hydrogen in NBR exposed to high-pressure hydrogen are reported.

\section{EXPERIMENTAL PROCEDURES}

\section{Materials}

Unfilled NBR (Nihon ZEON Nipol 1042) was employed as the experimental specimen after sulfur and vulcanization accelerators were added. The chemical composition and physical properties of the NBR sample are shown in Table 1. After mixing in the sulfur and accelerators, the composite was vulcanized at $170{ }^{\circ} \mathrm{C}$ for $10 \mathrm{~min}$. Assignment of each group in the chemical structure of NBR in the ${ }^{1} \mathrm{H}$ and ${ }^{13} \mathrm{C}$ NMR spectra was previously reported in detail; $;{ }^{18}$ the groups are shown in Figure 1 to represent the peaks of the spectra in this report.

\section{Exposure conditions}

Hydrogen exposure took place in a high-pressure hydrogen vessel filled with hydrogen $(99.999 \%)$ at $303 \mathrm{~K}$ under the following pressures: $10 \mathrm{MPa}, 20 \mathrm{MPa}$ and $100 \mathrm{MPa}$. The vessel was purged with nitrogen at $0.6 \mathrm{MPa}$ four times, and then with hydrogen at $0.6 \mathrm{MPa}$ for an additional four cycles before the exposure, and it was purged with nitrogen after the measurement.

\section{Instruments}

NMR measurements were performed using an AVANCE-III $500 \mathrm{MHz}$ : Bruker Biospin (Billerica, MA, USA) with a solution probe $(\phi 10 \mathrm{~mm})$ and three solid probes $(\phi 7, \phi 4$ and $\phi 2.5 \mathrm{~mm})$. The temperature was maintained at $303 \mathrm{~K}$ using a BCU-EXTREME. The time dependency of the spectra during hydrogen elimination was evaluated with a single pulse sequence. The hydrogen quantity was analyzed with a thermal desorption analyzer (TDA) JSH-201: J-SCIENCE LAB CO., LTD (JSL, Kyoto, Japan). ${ }^{16}$

\section{Procedures}

Measurement by NMR equipped with a solution probe. Gas eliminated from the specimens was analyzed using a solution probe after they were sealed in a $\phi$ 10-mm pressure-resistant NMR tube made of Pyrex (Nihon Seimitsu Kagaku Co. Ltd. N-10HP, Tokyo, Japan), as shown in Figure 2 (1). The typical silicon rubber seal for the NMR tube was replaced with NBR filled with carbon black to prevent hydrogen gas from leaking. The specimen $(4 \times 4 \times 60 \mathrm{~mm})$ was placed in the tube immediately after hydrogen exposure. The NMR measurement was performed under the conditions of $90^{\circ}$ single pulse (pulse width: $23 \mu \mathrm{s}$; pulse power: $34 \mathrm{~W}$; acquisition time: $6.87 \mathrm{~ms}$; observation center: 5.0 p.p.m.; sweep width: 20 p.p.m.; scan repetition: 64 times). Another pressure-resistant NMR tube made of Pyrex (Nihon Seimitsu Kagaku Co Ltd $\mathrm{N}-10 \mathrm{H}$ ), as shown in Figure 2 (2), was used for free hydrogen gas to determine its pressure dependency. Methanol- $\mathrm{d} 4$ with tetramethylsilane was placed in the capillary tube in the NMR tube for lock and shim optimization. The methanol that is partly protonated was used to measure the actual temperature of the filled gas in the tube. Hydrogen gas under pressures of $0.3-1.4 \mathrm{MPa}$ was filled into the tube at $303 \mathrm{~K}$ and measured using the solution probe. The NMR measurement was performed under the conditions of $90^{\circ}$ single pulse (pulse width: $23 \mu$ s; pulse power: $34 \mathrm{~W}$; acquisition time: $3.174 \mathrm{~s}$; observation center: 6.2 p.p.m.; sweep width: 20 p.p.m.; scan repetition: 128 times).

Table 1 Chemical composition (part by weight) and physical properties of vulcanized NBR

\begin{tabular}{lc}
\hline NBR (Nipol 1042) & 100 \\
Stearic acid & 1 \\
Zinc oxide & 5 \\
Sulfur & 1.5 \\
MBTS & 1.5 \\
TMTD & 0.5 \\
Hardness & $\mathrm{A} 52$ \\
Density (g $\mathrm{cm}^{-3}$ ) & 1.035 \\
Elastic modulus, $E$ (MPa) & 1.7 \\
Nominal fracture strength $\sigma \mathrm{n}(\mathrm{MPa})$ & 2.0 \\
Stretch at fractur, $\lambda \mathrm{F}$ & 4.3 \\
Crosslinking density (mol per cc) & $3.1 \times 10^{3}$
\end{tabular}

Abbreviations: MBTS, 2-benzothiazolyl disulfide; TMTD, tetramethylthiuram disulfide.

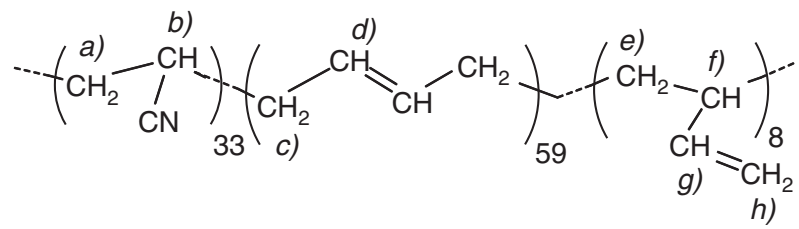

Figure 1 Scheme of NBR and the assignment of groups.

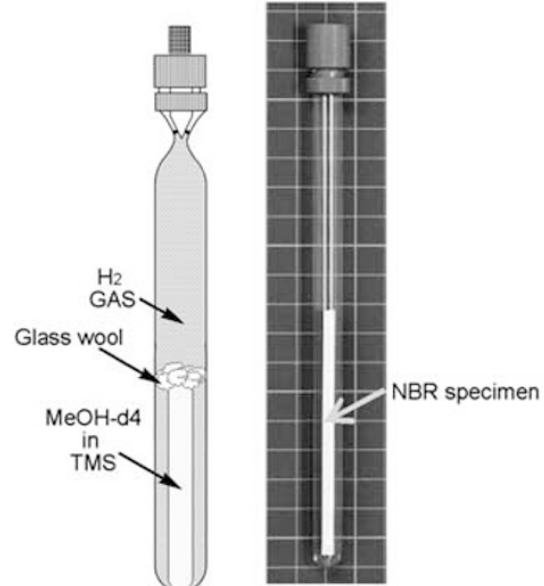

(1)

(2)

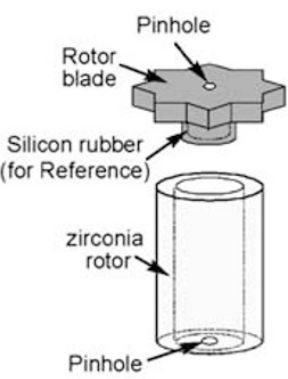

(3)
Figure 2 Photograph and sketch of the instrument for NMR measurement. (1) Pressure-resistant NMR tube for sealing in free hydrogen gas with the reference material, tetramethylsilane in methanol- $\mathrm{d}_{4}$, in the inner capillary tube. (2) Sealable pressure-resistant NMR tube (containing an NBR specimen inside). (3) Zirconia rotor with pinholes on the top and bottom with silicon rubber filled in the rotor blade as a reference. A full color version of this figure is available at Polymer Journal online.

Measurement by NMR equipped with solid probes. Hydrogen dissolved in the specimens was analyzed using solid probes with 7 and $4 \mathrm{~mm}$ zirconia rotors with MAS speeds of 8 and $15 \mathrm{KHz}$, respectively. The zirconia rotors were prepared by making pinholes both on the top and the bottom to release hydrogen gas and then filling the rotor blade with silicon rubber for the reference of the chemical shift and the quantification of hydrogen in the rubber to make the measurement conditions equal (as shown in Figure 2 (3)). The specimens of $\phi 4 \times 12, \phi 2.8 \times 12$ and $\phi 1.3 \times 9 \mathrm{~mm}$ were filled in the 7,4 and $2.5 \mathrm{~mm}$ rotors, respectively, and then were exposed to hydrogen. The NMR measurement was performed under the conditions of a $90^{\circ}$ single pulse $(7 \mathrm{~mm}$; pulse width: $5.0 \mu \mathrm{s}$; pulse power: $35 \mathrm{~W}$; acquisition time: $328 \mathrm{~ms})(4 \mathrm{~mm}$; pulse 
width: $5.0 \mu \mathrm{s}$; pulse power: $35 \mathrm{~W}$; acquisition time: $547 \mathrm{~ms})(2.5 \mathrm{~mm}$; pulse width: $2.5 \mu$ s; pulse power: $37 \mathrm{~W}$; acquisition time: $547 \mathrm{~ms}$ ). The observation center was at 5.0 p.p.m., whereas the sweep width was 20 p.p.m.; the scan repetition was performed 64 times.

\section{RESULTS AND DISCUSSION}

Measurement conditions for hydrogen-exposed rubber materials To analyze the dissolved hydrogen molecules in the rubber materials, it was important to determine suitable measurement conditions with respect to the damage of the sample due to the high-speed rotation of the MAS condition. Figure 3 shows the comparison of the ${ }^{1} \mathrm{H}$ NMR spectra of NBR in the solid state. In general, when high-speed MAS is applied, NMR affords higher resolution of the spectra. However, there are some disadvantages in the application of the MAS technique at speeds as high as $35 \mathrm{kHz}$, causing the extraction of dissolved hydrogen molecules from the exposed rubber samples due to the increased pressure on the sample by the acceleration of rotation. Pressurization caused by higher MAS speeds and downsizing of the samples accelerated the elimination of detectable hydrogen from the samples. Considering those benefits and disadvantages, the MAS frequency for the measurements of hydrogen-containing rubber samples was selected to be $8 \mathrm{kHz}$ and $15 \mathrm{kHz}$. Figure 3 (1) shows the ${ }^{1} \mathrm{H}$ NMR spectrum of NBR in the solid state using the NMR equipped with a solution probe. The resolution of the spectrum is relatively low compared with that of the spectrum obtained by the solid-state probe; however, this method was able to obtain the signals from the eliminated gaseous phase hydrogen molecules and the NBR sample itself in the same sample tube simultaneously.

\section{Measurements of gaseous phase hydrogen (free hydrogen gas)}

For the detailed analysis of dissolved hydrogen, the effect of gas pressure on the chemical shift of gaseous phase hydrogen was evaluated. We have previously reported the chemical shift of free hydrogen ${ }^{19}$ and detailed its temperature dependency and pressure dependency. ${ }^{20}$ The result of that study is mentioned briefly in this paper. The pressure dependency of the ${ }^{1} \mathrm{H}$ NMR spectra of free hydrogen gas in the pressure range of $0.3-1.4 \mathrm{MPa}$ at $303 \mathrm{~K}$ is shown in Figure 4. The temperature of each measurement was estimated to be $303 \pm 0.1 \mathrm{~K}$ by comparing the chemical shifts of the methyl group and hydroxyl group of methanol. ${ }^{21}$ The margin of error for the chemical shift was calculated to be $<0.00016$ p.p.m. from the result of the temperature dependency examination. ${ }^{22}$ The relationship between the filled gas pressure and the chemical shift of hydrogen gas is shown in Figure 5. The chemical shift of free hydrogen gas at $303 \mathrm{~K}$ was ensured to show an upfield shift in accordance with pressure rise. The value of the shift at $303 \mathrm{~K}$ can be denoted by equation (1):

$$
\delta \mathrm{H}_{2}=-0.0669 \mathrm{P}+7.29
$$

where $\mathrm{P}$ is the filled gas pressure measured with a Bourdon gage (MPa) and $\delta \mathrm{H}_{2}$ is the chemical shift (p.p.m. from tetramethylsilane) of gaseous hydrogen. The intercept of this equation, 7.29 p.p.m., is considered to be the chemical shift of gaseous hydrogen in atmospheric pressure $(0.1 \mathrm{MPa}$, absolute pressure) at $303 \mathrm{~K}$.

\section{Determination of eliminated hydrogen from rubber using a solution probe}

${ }^{1} \mathrm{H}$ spectra obtained with a solution probe of a specimen exposed to $100 \mathrm{MPa}$ of hydrogen and then sealed in pressure-resistant NMR tube are shown in Figure 6. Two peaks at approximately 1.8 and 5.5 p.p.m. in the spectra of the unexposed specimen (1-b) were assigned to the ${ }^{1} \mathrm{H}$ in the NBR structure, based on the solution spectra of crude

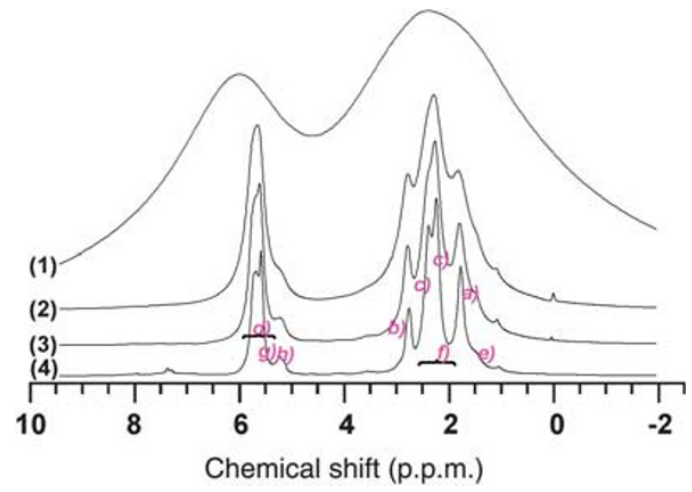

Figure 3 Differences in the resolution of the ${ }^{1} \mathrm{H}$ NMR spectra of solid-state NBR. (1) Spectra of solid-state NBR in a sealed sample tube equipped with solution probe. Spectra of solid-state NBR with MAS probe and; (2) MAS speed $8 \mathrm{kHz}$; (3) MAS speed $15 \mathrm{kHz}$; or (4) MAS speed $35 \mathrm{kHz}$.

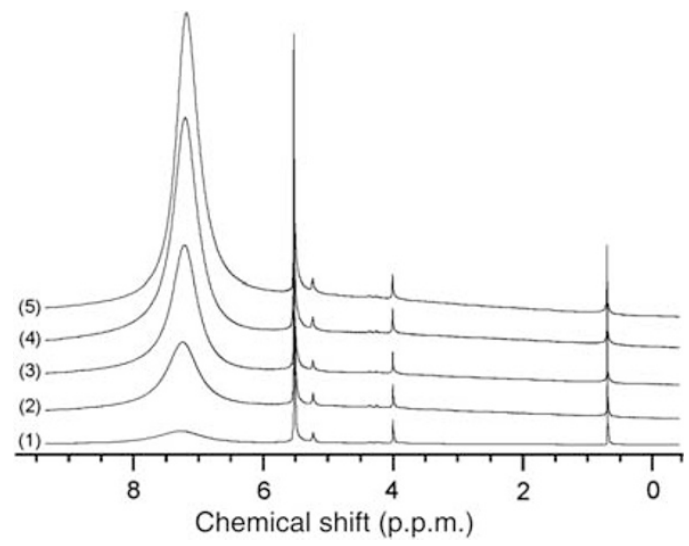

Figure $4^{1} \mathrm{H}$ NMR spectra of free hydrogen gas showing pressure dependency at $303 \mathrm{~K}$. Spectra of free hydrogen gas in the pressure range of: (1) $0.2 \mathrm{MPa}$, (2) $0.6 \mathrm{MPa}$, (3) $1.0 \mathrm{MPa}$, (4) $1.2 \mathrm{MPa}$ and (5) $1.4 \mathrm{MPa}$.

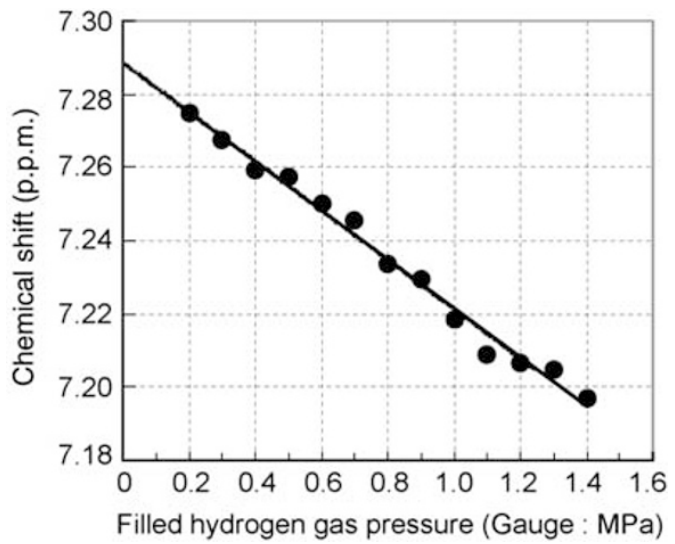

Figure 5 Relationship between pressure and the chemical shift of gaseous hydrogen at $303 \mathrm{~K}$

unexposed NBR (1-a). The spectrum of the specimen maintained for $5.5 \mathrm{~h}$ in the tube after exposure (1-c) also shows peaks with the same chemical shifts as the unexposed one. Aside from those peaks, a 

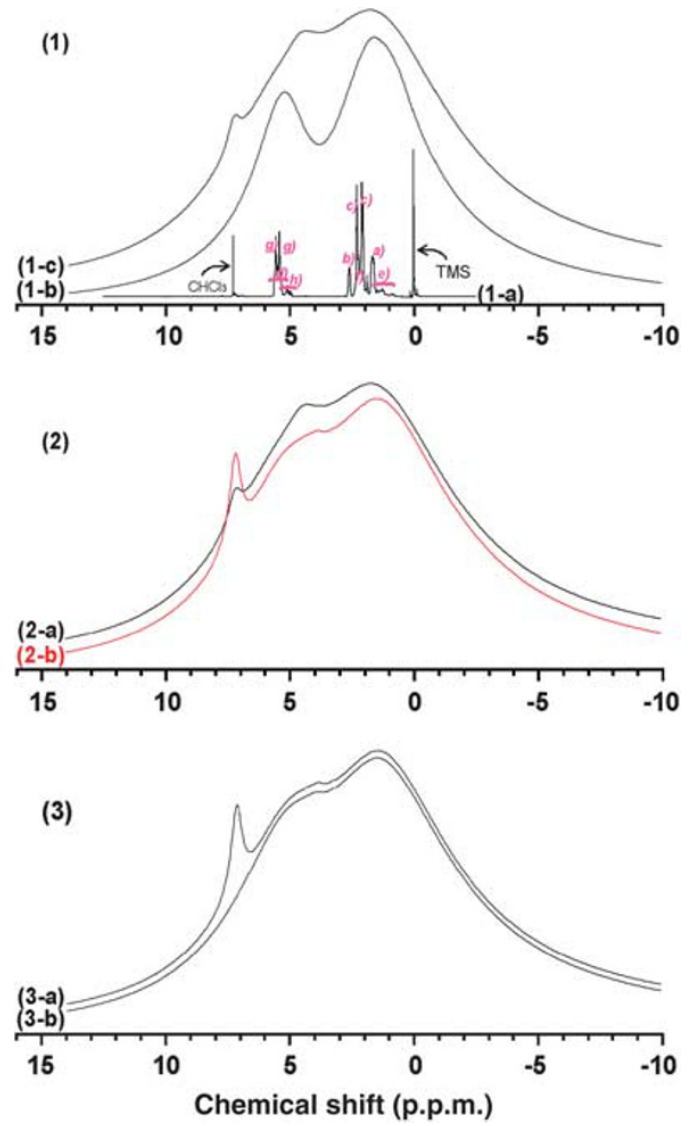

Figure $6{ }^{1} \mathrm{H}$ NMR spectra of NBR specimens exposed to $100 \mathrm{MPa}$ hydrogen gas at $303 \mathrm{~K}$ for $24 \mathrm{~h}$ in a sealed sample tube equipped with a solution probe. (1-a) Liquid-phase ${ }^{1} \mathrm{H}$ NMR spectra of crude NBR in $\mathrm{CDCl}_{3}$. (1-b) Spectrum of unexposed specimen. (1-c) and (2-a) Samples kept for $5 \mathrm{~h}$ with surrounding gas in a sealed sample tube. (2-b) and (3-a) Samples kept for $12.5 \mathrm{~h}$ with surrounding gas in a sealed sample tube. (3-b) Spectrum of the NBR specimen after removing the surrounding gas by unsealing the sample tube.

shoulder peak at approximately 7.2 p.p.m. appeared in the spectrum, which is not derived from rubber. In the spectrum of the specimen kept for $12.5 \mathrm{~h}$ in the tube after exposure (2-b), the peak at 7.2 p.p.m. significantly grows, whereas the peak at approximately 4.6 p.p.m. decreases. At this point of time, the tube was unsealed, and NMR measurement was conducted. In the spectrum of the specimen that was taken immediately after being unsealed, the peak at 7.2 p.p.m. disappeared, whereas the rest of the spectrum did not change. It can be explained that the peak at 7.2 p.p.m. represents the eliminated gas because it increased as time passed and then disappeared when the tube was unsealed to release the gas surrounding the specimen. The peak was assigned to free hydrogen gas based on the chemical shift of free hydrogen gas at 7.29 p.p.m., as mentioned above. The broad line shape with a peak at 7.2 p.p.m., assigned to free hydrogen gas, is similar to that of hydrogen in the rubber structure. The broadening agrees with the fact that the resonance lines of small symmetric molecules, such as hydrogen and methane, which exhibit extremely fast relaxation, are broader than for liquids. ${ }^{1}$ Additionally, the spectrum at approximately 4.6 p.p.m. was supposed to be attributed to the dissolved hydrogen in NBR; the intensity of the spectrum decayed due to the decrease of hydrogen quantity by elimination with time after exposure. The significant changes of these peaks suggest

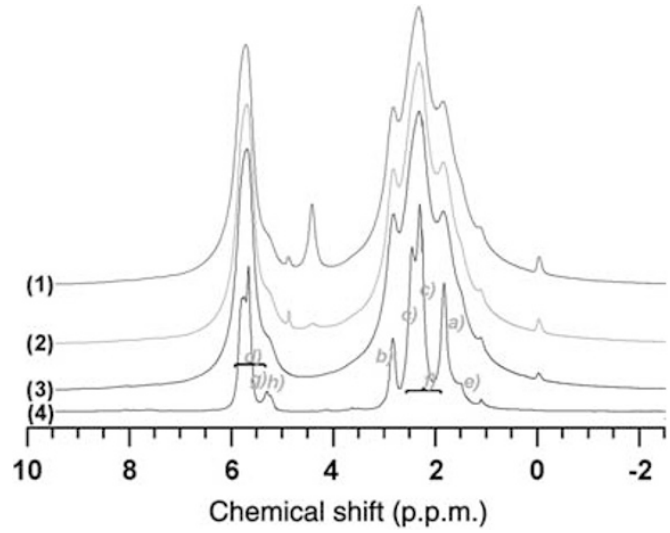

Figure 7 Solid-state ${ }^{1} \mathrm{H}$ NMR spectra of NBR original samples and NBR after hydrogen exposure. (1) After $1 \mathrm{~h} 100 \mathrm{MPa}$ exposure (MAS speed of $8 \mathrm{kHz}$ ). (2) After $1 \mathrm{~h} 20 \mathrm{MPa}$ exposure (MAS speed of $8 \mathrm{kHz}$ ). (3) Unexposed original samples (MAS speed of $8 \mathrm{kHz}$ ). (4) Unexposed original samples (MAS speed of $35 \mathrm{kHz}$ ). A full color version of this figure is available at Polymer Journal online.

that the elimination of dissolved hydrogen molecules in the specimen transferred to free hydrogen gas were eliminated as gaseous hydrogen.

Additionally, the dissolved hydrogen in NBR was supposed to be observed at approximately 4.6 p.p.m. because the peak intensity decreased as time passed.

\section{Exposure pressure dependency of dissolved hydrogen in NBR}

The measurements of the exposed specimens using the solid-state MAS probe are shown in Figure 7, along with spectrum of the unexposed specimen. The spectra taken at an MAS speed of $8 \mathrm{kHz}$ shows peaks at 1.9, 2.3, 2.8 and 5.7 p.p.m. and a shoulder peak at 5.3 p.p.m. Those peaks were attributed to the NBR structure, referencing the spectrum of unexposed NBR using high-resolution measurement at an MAS speed of $35 \mathrm{kHz}$. In both spectra of samples exposed to 100 and $20 \mathrm{MPa}$, peaks were detected at 4.3 p.p.m. (represented by [A]) and 4.8 p.p.m. ([B]) with the same chemical shifts, which were not assigned to the NBR structure. $[A]$ and $[B]$ were suggested not to have been derived from free hydrogen gas eliminated from the specimen nor remained in the rotors because their chemical shifts were different from free hydrogen gas at approximately 7.3 p.p.m. The intensity of peaks [A] and [B] decreased with time and their chemical shifts were similar to those at approximately 4.6 p.p.m. Same phenomena are seen in the measurement using pressure-resistant tube as shown in (2-a) and (2-b) in Figure 6 . We assumed that those peaks corresponded to hydrogen existing in the rubber specimen due to hydrogen exposure. Hydrogen quantity in the specimen exposed to $20 \mathrm{MPa}$ was calculated from the ratios of the total area of $[\mathrm{A}]$ and $[\mathrm{B}]$ to the peaks derived from the proton mole content of silicon rubber, of which the area of the peaks remains constant regardless of time because the silicon rubber was not exposed. In the previous report, ${ }^{16}$ the quantity of dissolved hydrogen in the rubber specimen after high-pressure hydrogen exposure was determined using TDA, and it was suggested that the eliminated gas was hydrogen in the gaseous phase.

Gaseous phase hydrogen contains two types of molecules, ortho and para hydrogen. Because para hydrogen is NMR-inactive, ${ }^{23}$ the para hydrogen molecule has a spin of zero; the ortho hydrogen molecule has a spin of one. In the quantitative estimation of the hydrogen molecule by NMR, the estimated quantity should be corrected in terms of the ortho and para ratio of hydrogen. There 


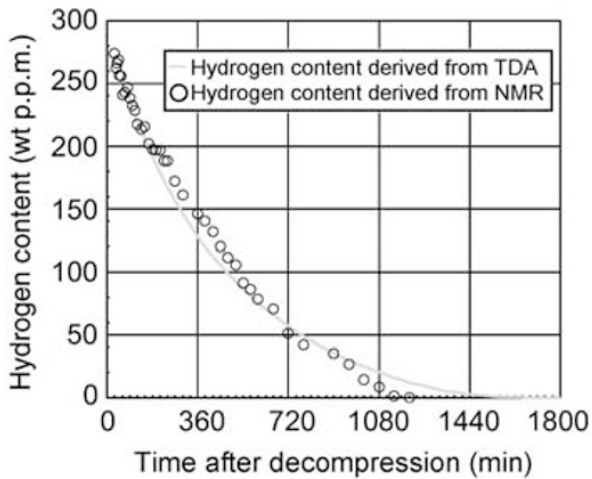

Figure 8 Isothermal profile of hydrogen content in the specimen exposed to $20 \mathrm{MPa}$ hydrogen gas during the elimination process measured by NMR and TDA at $303 \mathrm{~K}$. A full color version of this figure is available at Polymer Journal online.

is a report that the ratio of ortho and para hydrogen is constant at 3:1 above $273 \mathrm{~K} .{ }^{24}$ As the ortho and para ratio of hydrogen can be regarded as constant during NMR measurements at $303 \mathrm{~K}$, the appropriate estimates of hydrogen content are obtained. The time dependency of hydrogen quantification measured by NMR is shown in Figure 8 , along with the values of hydrogen quantity simultaneously measured by TDA. The result showed good agreement and suggest that NMR can determine all the hydrogen in the rubber material. It is necessary to disprove the possibility of $[A]$ and $[B]$ as gas existing in a blister. If hydrogen is in the bubble of a blister, the characteristics of the hydrogen should mimic free hydrogen gas, and the pressure should be lower than exposure pressure of $20 \mathrm{MPa}$. The chemical shift of free hydrogen gas exposed to $20 \mathrm{MPa}$ was calculated to be 5.95 p.p.m. using equation (1). This means that the signal for the free hydrogen gas in the blister should show a low field shift from 5.59 p.p.m. when exposed to $20 \mathrm{MPa}$ of hydrogen gas. The peaks [A] (4.3 p.p.m.) and [B] (4.8 p.p.m.) were observed with chemical shifts smaller than 5.59 p.p.m., that is, in a higher field compared with free hydrogen gas. Therefore, $[\mathrm{A}]$ and $[\mathrm{B}]$ do not represent free hydrogen in a blister.

Furthermore, the ratio of $[\mathrm{A}]$ to $[\mathrm{B}]$ increases as the exposure pressure increases from 10 to $100 \mathrm{MPa}$. The isothermal pressure dependency of hydrogen quantities [A] and [B] $1 \mathrm{~h}$ after the hydrogen exposure is shown in Figure 9. The ratio of $[\mathrm{A}]$ to $[\mathrm{B}]$ increased by the increase of exposure pressure. It was suggested that sorption sites in the material did not increase with the exposure pressures in this study up to $100 \mathrm{MPa}$, as the cross-link density or chemical structure of the exposed material did not change. ${ }^{17}$ Thus, it may be reasonable to explain that the ratio change of $[\mathrm{A}]$ and $[\mathrm{B}]$ is not caused by the increase of sorption sites, but rather by the difference in the solubility of the hydrogen.

\section{Time dependency of the ${ }^{1} \mathrm{H}$ NMR spectra of dissolved hydrogen in NBR}

${ }^{1} \mathrm{H}$ MAS solid-state NMR was employed to evaluate the hydrogen in the rubber in detail. The results of NMR measurements at an MAS speed of $15 \mathrm{kHz}$ over a period from 1 to $24 \mathrm{~h}$ after the specimen was decompressed after $100 \mathrm{MPa}$ hydrogen exposure is shown in Figure 10. Peaks at 1.9, 2.3, 2.8 and 5.7 p.p.m. and a shoulder peak at 5.3 p.p.m. were observed, and all the peaks were assigned to the rubber structure. Peaks attributed to dissolved hydrogen were observed at 4.3 p.p.m. [A] and 4.8 p.p.m. [B], similar to the spectra

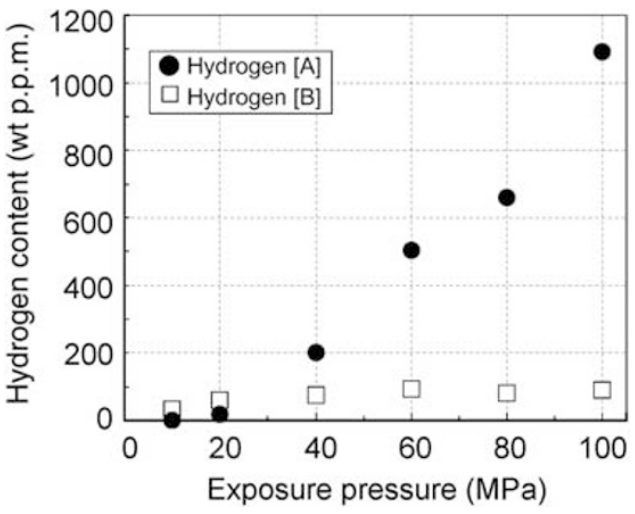

Figure 9 Influence of exposure pressure on hydrogen content from 10 to $100 \mathrm{MPa}$, and $1 \mathrm{~h}$ after exposure at $303 \mathrm{~K}$.

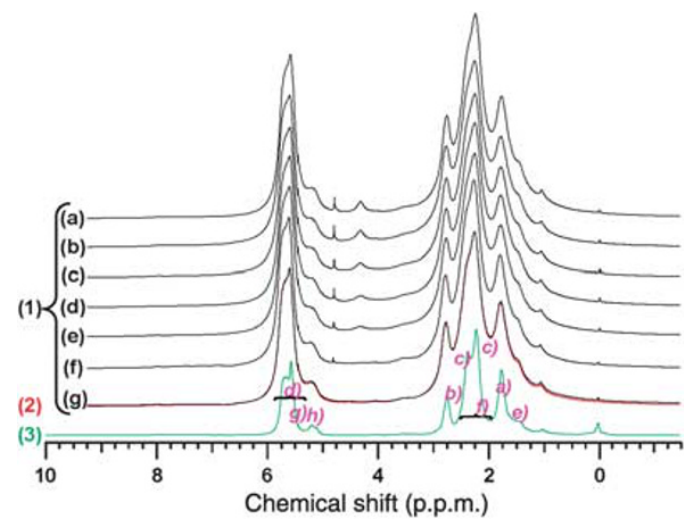

Figure 10 Solid-state ${ }^{1} \mathrm{H}$ NMR spectra of NBR original samples and NBR after hydrogen exposure at $303 \mathrm{~K}$. (1) Solid-state ${ }^{1} \mathrm{H}$ NMR spectra of NBR specimens exposed to $100 \mathrm{MPa}$ hydrogen gas at $303 \mathrm{~K}$ for $24 \mathrm{~h}$ and after an additional (a) $1 \mathrm{~h}$, (b) $3 \mathrm{~h}$, (c) $7 \mathrm{~h}$, (d) $10 \mathrm{~h}$, (e) $14 \mathrm{~h}$, (f) $18 \mathrm{~h}$ and (g) $24 \mathrm{~h}$ from decompression (MAS speed $15 \mathrm{kHz}$ ). (2) Solid-state ${ }^{1} \mathrm{H}$ NMR spectrum of an unexposed NBR specimen (MAS speed of $15 \mathrm{kHz}$ ). (3) Solid-state ${ }^{1} \mathrm{H}$ NMR spectrum of an unexposed NBR specimen (MAS speed of $35 \mathrm{kHz}$ ).

of $8 \mathrm{kHz}$ MAS NMR. The intensities of the peaks [A] and [B] decayed with time after decompression, and the decay ceased $24 \mathrm{~h}$ after decompression. The spectrum at this point of time overlapped with the unexposed sample. Any remaining hydrogen in the specimen $24 \mathrm{~h}$ after exposure was evaluated using TDA. The hydrogen quantity was measured to be zero, and the NMR spectra after TDA measurement overlapped with the unexposed line.

There was no change in the chemical shift with time. Thus, it was found that the characteristics of individual hydrogen do not change due to hydrogen elimination. The spin lattice relaxation times (T1) of [A] and [B] determined by the inversion recovery method were 0.08 and $0.49 \mathrm{~s}$, respectively, and their half-widths were 148 and $47 \mathrm{~Hz}$, respectively. Both values are significantly different from one another. Accordingly, it is suggested that the hydrogen dissolved in the rubber existed in two different forms with different mobilities.

The isothermal time dependency of the ratio of hydrogen $[\mathrm{A}]$ and [B] is shown in Figure 11. The ratio of [B] to the total quantity $([\mathrm{A}]+[\mathrm{B}])$ significantly increased with time when the total quantity of hydrogen in the rubber decreases. Moreover, as mentioned above (Figure 9), hydrogen [A] becomes more dominant than [B] as the exposure pressure increased. This phenomenon can be interpreted as 


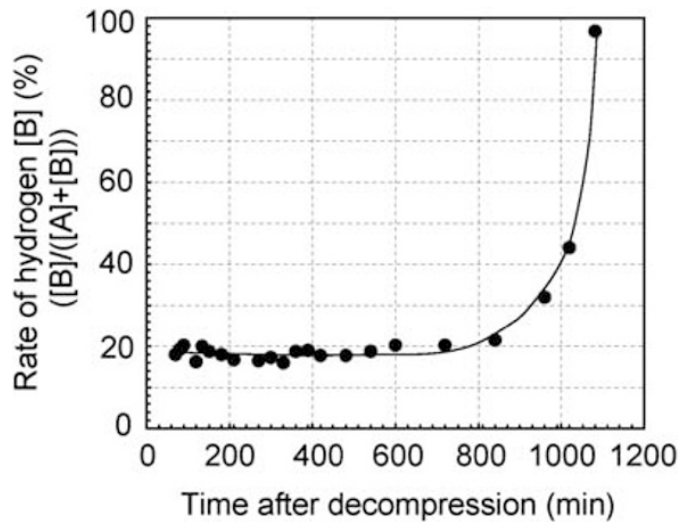

Figure 11 Time dependency of the ratio of hydrogen content $[A]$ and $[B]$ at $303 \mathrm{~K}$.

the difference of the mobility that may be caused by the difference in the strength of the interaction between the hydrogen molecules and rubber molecules in the cross-linked structures.

Water-containing polymeric materials, such as hydrogels, are considered to contain two kinds of water: free water and constrained water. ${ }^{25}$ Using this analogy of the hydrogels, hydrogen [A] with the peak at 4.5 p.p.m. and [B] at 4.8 p.p.m. are attributed to the hydrogen constrained to the molecular chain of the NBR and the hydrogen in the free volume of the NBR, respectively, because $[\mathrm{A}]$ has a significantly greater mobility than $[\mathrm{B}]$, and both hydrogens are dissolved hydrogens in NBR.

This result may support the explanation that [B] represents hydrogen in the free volume, which barely gains a shielding effect of rubber molecules. The phenomenon of change in the ratio of hydrogen $[\mathrm{A}]$ and $[\mathrm{B}]$ with increasing pressure may be interpreted as hydrogen transfers into the molecular chains of the NBR, where hydrogen barely penetrates under low pressure. It seems reasonable to think that the hydrogen transfers to the free volume during the elimination process; thus, hydrogen derived from free volume [B] becomes dominant at a later stage during the elimination process.

\section{CONCLUSION}

We successfully analyzed the characteristics and behavior of hydrogen dissolved in NBR with respect to high-pressure hydrogen exposure using ${ }^{1} \mathrm{H}$ NMR. The NMR spectra indicated two peaks at 4.5 and 4.8 p.p.m., and each $\mathrm{T} 1$ was 0.08 and 0.49 s, respectively. According to the hypothesis that the two peaks represented dissolved hydrogen molecules, hydrogen quantifications by TDA and NMR showed good correlation. Furthermore, these chemical shifts were ensured to be different from that of free hydrogen in a blister, based on the value derived from the pressure dependency of the chemical shift of gaseous phase hydrogen. The simultaneous evaluation of hydrogen in the rubber and eliminated gas using a pressure-resistant NMR tube clarified the forms of hydrogen present. Two kinds of hydrogen were observed, and they were both hydrogen dissolved in NBR but with different mobilities. The ratio of the two characteristic peaks of hydrogen was affected by exposure pressure and time during the elimination process.

Further analysis on the influence of the two kinds of hydrogen on the properties of rubber materials, such as swelling or destruction, and understanding the relationship between the concentration of dissolved hydrogen and the chemical structure of rubber or other polymers may contribute to the design of new materials that are resistant to high-pressure hydrogen. It may also be possible to utilize hydrogen, the smallest molecule, as a probe to analyze rubber or other compounds of higher order in future investigations.

\section{ACKNOWLEDGEMENTS}

This research was partly supported by the project 'Fundamental Research Project on Advanced Hydrogen Science (2006-2012)' of the New Energy and Industrial Technology Development Organization (NEDO), Japan. We thank Prof Tadashi Narita and Prof Hiroshi Hamana (Saitama Institute of Technology) for their generous guidance with the NMR measurements. We also thank late Mr Kazuhiko Yamada in Bruker BioSpin KK for their kind cooperation for installing of the NMR.

1 Suarez, C. Gas-phase NMR spectroscopy. Chem. Educ. 3, 1-7 (1998)

2 Conner, W. C., Weist, E. L., Ito, T. \& Fraissard, J. Characterization of the porous structure of agglomerated microspheres by xenon-129 NMR spectroscopy. J. Phys. Chem. 93, 4138-4142 (1989).

3 Nagano, J., Eguchi, T., Asanuma, T., Masui, H., Nakayama, H., Nakamura, N. \& Derouane, E. G. ${ }^{1} \mathrm{H}$ and ${ }^{129} \mathrm{Xe}$ NMR investigation of the microporous structure of dealuminated $\mathrm{H}$-mordenite probed by methane and xenon. Micro. Meso. Mater. 33, 249-256 (1999).

4 Miyoshi, T., Takegoshi, K. \& Terao, T. ${ }^{129}$ Xe NMR study of free volume and phase separation of the polystyrene/poly(vinyl methyl ether) blend. Polymer 38, 5475-5480 (1997).

5 Suzuki, T., Yoshimizu, H. \& Tsujita, Y. Analysis of gas transport properties of PPO/PS blends by ${ }^{129}$ Xe NMR spectroscopy. Polymer 44, 2975-2982 (2003).

6 Yoshimizu, H., Suzuki, T. \& Tsujita, Y. Characterization of polymer in the solid state by ${ }^{129}$ Xe NMR spectroscopy. Seni Gakkaishi 60, 26-31 (2004).

7 Yoshimizu, H., Tsukahara, M., Suzuki, T., Toida, J., Ando, A., Watanabe, J. \& Tsujita, Y. Layered structure and, Xe sorption and diffusion properties of low-density liquidcrystalline polyesters with n-alkyl side chains. J. mol. Struct. 739, 19-26 (2005).

8 Parker, Jr. W. O., Ferrando, A., Ferri, D. \& Canepari, V. Cross-link density of a dispersed rubber measured by ${ }^{129}$ Xe chemical shift. Macromolecules 40, 5787-5790 (2007).

9 Murata, M., Murata, Y. \& Komatsu, K. Synthesis and properties of endohedral $\mathrm{C}_{60}$ encapsulating molecular hydrogen. J. Am. Chem. Soc. 128, 8024-8033 (2006).

10 Shen, K. \& Pietrass, T. NMR spectroscopy of hydrogen adsorption on single-walled carbon nanotubes after exposure to hight pressure. Solid State Nucl. Magn. Reson. 29, 125-131 (2006).

11 Nishimura, S. \& Fujiwara, H. Detection of hydrogen dissolved in acrylonitrile butadiene rubber by ${ }^{1} \mathrm{H}$ nuclear magnetic resonance. Chem. Phys. Lett. 522, 43-45 (2012).

12 Briscoe, B. J., Savvas, T. \& Kelly, C. T. Explosive decompression failure of rubber. Rubber Chem. Technol. 67, 384-416 (1994).

13 Yamabe, J., Nakao, M., Fujiwara, H. \& Nishimura, S. In: Hydrogen Conference (Jackson Lake Lodge, USA, 2008).

14 Yamabe, J., Nakao, M., Fujiwara, H. \& Nishimura, S. Influence of fillers on hydrogen penetration properties and blister fracture of EPDM composites exposed to $10 \mathrm{MPa}$ hydrogen gas. Transact. Jpn Soc. Mech. Eng. 74, 971-981 (2008).

15 Yamabe, J., Koga, A. \& Nishimura, S. Durability and hydrogen permeation properties of sealing rubber under high pressure hydrogen gas. Nippon. Gomu. Kyokaishi $\mathbf{8 3}, 159$ 166 (2010)

16 Yamabe, J. \& Nishimura, S. Influence of fillers on hydrogen penetration properties and blister fracture of rubber composites for 0-ring exposed to high-pressure hydrogen gas. Int. J. Hydrogen Energy 34, 1977-1989 (2009).

17 Fujiwara, H., Yamabe, J. \& Nishimura, S. Evaluation of the change in chemical structure of acrylonitrile butadiene rubber after high-pressure hydrogen exposure. Int. J. Hydrogen Energy 37, 8729-8733 (2012).

18 Fujiwara, H., Yamabe, J. \& Nishimura, S. Determination of chemical structure of vulcanized NBR with solid state $1 \mathrm{H}, 13 \mathrm{C}$ NMR spectroscopy. Kobunshi Ronbunshu 66, 363-372 (2009).

19 Fujiwara, H., Yamabe, J. \& Nishimura, S. Determination of chemical shift of gas-phase hydrogen molecules by ${ }^{1} \mathrm{H}$ nuclear magnetic resonance. Chem. Phys. Lett. 498, 42-44 (2010).

20 Fujiwara, H. \& Nishimura, S. Pressure and temperature dependency for chemical shift in ${ }^{1} \mathrm{H}$ nuclear magnetic resonance spectra of gaseous phase hydrogen. Chem. Phys. Lett (in press).

21 Van Geet, A. L. Calibration of methanol nuclear magnetic resonance thermometer at low temperature. Anal. Chem. 42, 679-680 (1970).

22 Raidford, D. S., Fisk, C. L. \& Becker, E. D. Calibration of methanol and ethylene glycol nuclear magnetic resonance thermometers. Anal. Chem. 51, 2050-2051 (1979).

23 Matsumoto, M. \& Espenson, J. H. Kinetics of the interconversion of parahydrogen and orthohydrogen catalyzed by paramagnetic complex ions. J. Am. Chem. Soc. 127, 11447-11453 (2005)

24 Züttel, A., Borgschutle, A. \& Schlapbach, L. Hydrogen as a Future Energy Carrier (Wiley-VCH Verlag GmbH \& Co. KGaA, Weinheim, Germany, 2008).

25 Alesiani, M., Pirazzoli, I. \& Maraviglia, B. Factors affecting early-age hydration of ordinary portland cement studied by NMR: fineness, water-to-cement ratio and curing temperature. Appl. Magn. Reson. 32, 385-394 (2007). 